\title{
Completeness generated by convergence with respect to a $\sigma$-ideal
}

\author{
Marek Balcerzak \\ Technical University of Łódź, Poland
}

- Typeset by FoilTEX - 
It is well known that a sequence of real-valued measurable functions $\left(f_{n}\right)_{n \in \mathbb{N}}$ on $[0,1]$ converges in measure to a function $f$ if and only if each subsequence of $\left(f_{n}\right)_{n \in \mathbb{N}}$ contains a subsequence which converges to $f$ almost everywhere.

This fact was used by E. Wagner (1981) to define an abstract kind of convergence.

We assume that $\mathcal{S}$ is a $\sigma$-algebra of subsets of a given set $Y$ and $\mathcal{J}$ is a proper $\sigma$-ideal (i.e. $Y \notin \mathcal{J}$ ) of subsets of $Y$. First we assume additionally that $\mathcal{J} \subset \mathcal{S}$.

By $\mathcal{F}(\mathcal{S})$ we denote the set of all $\mathcal{S}$-measurable real-valued functions on $Y$. We say that a given property holds $\mathcal{J}$-almost everywhere on $Y$ if the set of all elements $y \in Y$ that do not have this property, belongs to $\mathcal{J}$.

A sequence $\left(f_{n}\right)_{n \in \mathbb{N}}$ of functions from $\mathcal{F}(\mathcal{S})$ is called convergent to $f \in \mathcal{F}(\mathcal{S})$ with respect to $\mathcal{J}$ if each subsequence of $\left(f_{n}\right)_{n \in \mathbb{N}}$ contains a subsequence convergent $\mathcal{J}$-almost everywhere to $f$. This is written as $f_{n} \stackrel{J}{\rightarrow} f$. 
In our considerations we might assume that functions from $\mathcal{F}(\mathcal{S})$ are defined $\mathcal{J}$-almost everywhere and have values in $\mathbb{R} \cup\{-\infty,+\infty\}$ but are finite $\mathcal{J}$-almost everywhere. This however does not lead to essentially more general situation, since using the respective equivalence relation, we come back to the case of finite valued functions defined everywhere.

Note that a limit of a sequence $\left(f_{n}\right)_{n \in \mathbb{N}}$ in $\mathcal{F}(\mathcal{S})$ convergent with respect to $\mathcal{J}$ is not uniquely determined, however any two limits are $\mathcal{J}$-a.e. equal on $Y$.

Denote by $\mathbb{N}^{\uparrow}$ the set of all increasing sequences of positive integers. Let $[E]^{<\kappa},[E]^{\leq \kappa}$ denote the families of subsets of $E$ of cardinality $<\kappa$ and $\leq \kappa$, respectively.

The following classical example is in a sense, universal since the same functions $f, f_{n}, n \in \mathbb{N}$, in $\mathcal{F}(\mathcal{S})$ witness that convergence " $\stackrel{\mathcal{J}}{\rightarrow}$ " need not imply convergence " $\rightarrow, \mathcal{J}$-a.e.", for a large class of pairs $(\mathcal{S}, \mathcal{J})$ on $[0,1]$. 
Example 1. Let $Y:=[0,1]$ and write the functions of the sequence

$$
\chi_{[0,1 / 2]}, \chi_{[1 / 2,1]}, \chi_{[0,1 / 4]}, \chi_{[1 / 4,1 / 2]}, \chi_{[1 / 2,3 / 4]}, \chi_{[3 / 4,1]}, \chi_{[0,1 / 8]}, \ldots
$$

as $f_{1}, f_{2}, f_{3}, \ldots$ Let $f \equiv 0$ on $[0,1]$. Then $f_{n} \rightarrow f$ in measure but $f_{n}(x) \rightarrow f(x)$ is false, for all $x \in[0,1]$. Clearly, the functions $f_{n}, n \in \mathbb{N}$, are Borel measurable. Let $\mathcal{S}$ be any $\sigma$-algebra on $[0,1]$ containing Borel sets. Observe that $f_{n} \stackrel{\mathcal{J}}{\rightarrow} f$ where $\mathcal{J} \subset \mathcal{S}$ is any proper $\sigma$-ideal containing all countable subsets of $Y$. Indeed, if $\left(n_{k}\right) \in \mathbb{N}^{\uparrow}$ then we can find $\left(k_{p}\right) \in \mathbb{N}^{\uparrow}$ such that $f_{n_{k_{p}}} \rightarrow f$ everywhere except for at most one point from $Y$.

Due to the Riesz theorem, convergence in measure on $[0,1]$ is equivalent to the respective Cauchy condition - if it is satisfied, a sequence of measurable functions is called fundamental in measure. 
It was observed by E. Wagner and W. Wilczyński (1999) that the Cauchy condition in measure can be described without the use of measure. This led to the notion of a sequence fundamental in category (when one uses as $\mathcal{J}$ the $\sigma$-ideal of meager subsets of $[0,1])$.

The result by $\mathrm{E}$. Wagner and W. Wilczyński states that a sequence of functions with the Baire property is convergent in category on $[0,1]$ (i.e. convergent with respect to the $\sigma$-ideal of meager sets) if and only if it is fundamental in category.

One can easily extend the those considerations to a general case.

Namely, we say that a sequence $\left(f_{n}\right)_{n \in \mathbb{N}}$ in $\mathcal{F}(\mathcal{S})$ is $\mathcal{J}$-Cauchy (or $\mathcal{J}$ fundamental) if, for any $\left(m_{k}\right)_{k \in \mathbb{N}},\left(n_{k}\right)_{k \in \mathbb{N}} \in \mathbb{N}^{\uparrow}$, the sequence $\left(f_{m_{k}}-f_{n_{k}}\right)_{k \in \mathbb{N}}$ converges, with respect to $\mathcal{J}$, to the constant function equal to zero on $Y$. Observe that $\left(f_{n}\right)$ is $\mathcal{J}$-Cauchy if and only if for any $\left(m_{k}\right),\left(n_{k}\right) \in \mathbb{N}^{\uparrow}$ there is $\left(k_{p}\right) \in \mathbb{N}^{\uparrow}$ such that $\left(f_{m_{k_{p}}}-f_{n_{k_{p}}}\right)_{p \in \mathbb{N}}$ tends to zero $\mathcal{J}$-a.e. on $Y$. 
Proposition 2. For every sequence $\left(f_{n}\right)$ of functions from $\mathcal{F}(\mathcal{S})$, if $\left(f_{n}\right)$ converges with respect to $\mathcal{J}$ to a function $f \in \mathcal{F}(\mathcal{S})$ then $\left(f_{n}\right)$ is a $\mathcal{J}$-Cauchy sequence.

If every $\mathcal{J}$-Cauchy sequence in $\mathcal{F}(\mathcal{S})$ is convergent, with respect to $\mathcal{J}$, to a function $f \in \mathcal{F}(\mathcal{S})$ (i.e. the converse of implication from Proposition 2 holds), we say that $\mathcal{F}(\mathcal{S})$ is $\mathcal{J}$-complete.

Example 3. By $\{\emptyset\}_{Y}$ we denote the $\sigma$-ideal on $Y$ consisting of $\emptyset$. Put $\mathcal{J}:=\{\emptyset\}_{Y}$ and let $\mathcal{S}$ be an arbitrary $\sigma$-algebra on $Y$. Then $f_{n} \stackrel{\jmath}{\rightarrow} f$ is equivalent to the usual pointwise convergence $f_{n} \rightarrow f$. Similarly, $\left(f_{n}\right)_{n \in \mathbb{N}}$ is $\mathcal{J}$-Cauchy if and only if $\left(f_{n}(x)\right)_{n \in \mathbb{N}}$ is a Cauchy sequence for every $x \in Y$. Hence we conclude that $\mathcal{F}(\mathcal{S})$ is $\mathcal{J}$-complete, by the completeness of $\mathbb{R}$. 
Let $\mathcal{M}$ and $\mathcal{N}$ stand for the $\sigma$-ideals of meager sets in $\mathbb{R}$ and of Lebesgue null sets in $\mathbb{R}$. Their restrictions to an interval $I \subset \mathbb{R}$ will be denoted by $\mathcal{M}_{I}$ and $\mathcal{N}_{I}$.

Let BAIRE and LEB stand for the $\sigma$-algebras of subsets of $\mathbb{R}$ with the Baire property and of Lebesgue measurable subsets of $\mathbb{R}$, respectively. Their restrictions to $I$ will be written as $\operatorname{BAIRE}_{I}$ and $\operatorname{LEB}_{I}$. Symbols $\mathcal{M}(Z)$ and $\operatorname{BAIRE}(Z)$ have the same meanings as $\mathcal{M}$ and BAIRE but they concern an uncountable Polish space $Z$ taken in place of $\mathbb{R}$.

Fact 4. - $\mathcal{F}\left(\mathrm{LEB}_{[0,1]}\right)$ is $\mathcal{N}_{[0,1]}$-complete (the classical Riesz theorem);

- $\mathcal{F}\left(\mathrm{BAIRE}_{[0,1]}\right)$ is $\mathcal{M}_{[0,1]}$-complete [WBW 1999];

- $\mathcal{F}\left(\operatorname{LEB}_{[0,1]} \cap \operatorname{BAIRE}_{[0,1]}\right)$ is $\mathcal{N}_{[0,1]} \cap \mathcal{M}_{[0,1]}$-complete [WBW 1999]. 
We know only one example of non- $\mathcal{-}$-completeness. Consider the partial order $\leq^{*}$ on $\mathbb{N}^{\uparrow}$ defined as follows: $\left(m_{j}\right) \leq^{*}\left(n_{j}\right)$ if there exists $k \in \mathbb{N}$ such that $m_{j} \leq n_{j}$ for all $j \geq k$.

A set $T \subset \mathbb{N}^{\uparrow}$ is called bounded if there exists $\left(n_{j}\right) \in \mathbb{N}^{\uparrow}$ such that $\left(m_{j}\right) \leq^{*}\left(n_{j}\right)$ for all $\left(m_{j}\right) \in T$. Let $\mathfrak{b}$ denote the minimal cardinality of an unbouned subset of $\mathbb{N}^{\uparrow}$, and let $\mathfrak{c}:=|\mathbb{R}|$.

It is known that $\omega_{1} \leq \mathfrak{b} \leq \mathfrak{c}$ and each of the conditions $\omega_{1}=\mathfrak{b}<\mathfrak{c}$, $\omega_{1}<\mathfrak{b}<\mathfrak{c}, \omega_{1}<\mathfrak{b}=\mathfrak{c}$ is consistent. Obviously the Continuum Hypothesis $(\mathrm{CH})$ implies $\mathfrak{b}=\mathfrak{c}$.

Fact 5. [Vladimirov 1960] $\mathcal{F}(\mathcal{P}(\mathfrak{b}))$ is not $[\mathfrak{b}]^{<\mathfrak{b}}$-complete.

Let $\mathcal{S}$ and $\mathcal{J}$ be a $\sigma$-algebra and a $\sigma$-ideal on $Y$. Note that convergence with respect to $\mathcal{J}$, and also $\mathcal{J}$-Cauchy condition and $\mathcal{J}$-completeness, defined before, make sense without assuming $\mathcal{J} \subset \mathcal{S}$. 
Namely, put

$$
\mathcal{J} \mid \mathcal{S}:=\{A \subset Y:(\exists B \in \mathcal{J} \cap \mathcal{S}) A \subset B\} .
$$

If $f, f_{n} \in \mathcal{F}(\mathcal{S}), n \in \mathbb{N}$, observe that " $f_{n} \rightarrow f$, $\mathcal{J}$-a.e.," is the same as the statement " $f_{n} \rightarrow f, \mathcal{J}\lceil\mathcal{S}$-a.e.".

Hence it follows that $f_{n} \stackrel{\mathcal{J}}{\rightarrow} f$ is equivalent to $f_{n} \stackrel{\mathfrak{J} \mathcal{S}}{\rightarrow} f$. So, our extended definition has been reduced to the case of the pair $(\mathcal{S}, \mathcal{J}\lceil\mathcal{S})$ with $\mathcal{J}\lceil\mathcal{S} \subset \mathcal{S}$. Similarly, we interpret the extended notions of a $\mathcal{J}$-Cauchy sequence and $\mathcal{J}$-completeness of $\mathcal{F}(\mathcal{S})$.

We study several operations preserving $\mathcal{J}$-completeness.

If a $\sigma$-algebra $\mathcal{S}$ on $Y$ is fixed, we say that two $\sigma$-ideals $\mathcal{J}_{1}$ and $\mathcal{J}_{2}$ on $Y$ are $\mathcal{S}$-orthogonal, if there is $A \in \mathcal{S}$ such that $A \in \mathcal{J}_{1}$ and $Y \backslash A \in \mathcal{J}_{2}$. 
Proposition 6. Assume that $\mathcal{S}_{i}$ is a $\sigma$-algebra and $\mathcal{J}_{i}$ is a $\sigma$-ideal on $Y$ for $i \in M$ where $M \neq \emptyset$. Let $\mathcal{J}=\bigcap_{i \in M} \mathcal{J}_{i}$ and $\mathcal{S}=\bigcap_{i \in M} \mathcal{S}_{i}$. We then have:

(a) $\mathcal{J}$ is a $\sigma$-ideal and $\mathcal{S}$ is a $\sigma$-algebra on $Y$.

(b) If $M$ is countable, $\mathcal{J}_{i} \uparrow \mathcal{S}=\mathcal{J}_{i}$ for $i \in M$ and $\mathcal{F}\left(\mathcal{S}_{i}\right)$ is $\mathcal{J}_{i}$-complete for $i \in M$, then $\mathcal{F}(\mathcal{S})$ is $\mathcal{J}$-complete.

(c) If $M$ is countable, $\mathcal{S}_{i}=\mathcal{S}$ and $\mathcal{J}_{i} \uparrow \mathcal{S}=\mathcal{J}_{i}$ for $i \in M$, and $\mathcal{J}_{i}, i \in M$, are pairwise $\mathcal{S}$-orthogonal, then $\mathcal{F}(\mathcal{S})$ is $\mathcal{J}$-complete if and only if $\mathcal{F}(\mathcal{S})$ is $\mathcal{J}_{i}$-complete for all $i \in M$. 
Proposition 7. For $M \neq \emptyset$, let $\left(Y_{i}\right)_{i \in M}$ be a family of pairwise disjoint sets, and for each $i \in M$, assume that $\mathcal{J}_{i}$ and $\mathcal{S}_{i}$ are a $\sigma$-ideal and a $\sigma$-algebra on $Y_{i}$. Put $Y:=\bigcup_{i \in M} Y_{i}, \underset{i \in M}{\oplus} \mathcal{J}_{i}:=\left\{A \subset Y:(\forall i \in M) A \cap Y_{i} \in \mathcal{J}_{i}\right\}$ and $\underset{i \in M}{\oplus} \mathcal{S}_{i}:=\left\{A \subset Y:(\forall i \in M) A \cap Y_{i} \in \mathcal{S}_{i}\right\}$. We then have:

(a) $\underset{i \in M}{\oplus} \mathcal{J}_{i}$ is a $\sigma$-ideal and $\underset{i \in M}{\oplus} \mathcal{S}_{i}$ is a $\sigma$-algebra on $Y$.

(b) If $M$ is countable, then $\mathcal{F}\left(\mathcal{S}_{i}\right), i \in M$, are $\mathcal{J}_{i^{-}}$complete iff $\mathcal{F}\left(\underset{i \in M}{\oplus} \mathcal{S}_{i}\right)$ is $\underset{i \in M}{\oplus} \mathcal{J}_{i^{-}}$ complete.

Corollary 8. The space $\mathcal{F}(\mathrm{LEB})$ is $\mathcal{N}$-complete. 
Proof. We have

$$
\mathcal{N}=\underset{m \in \mathbb{Z}}{\oplus} \mathcal{N}_{[m, m+1)} \text { and LEB }=\underset{m \in \mathbb{Z}}{\oplus} \operatorname{LEB}_{[m, m+1)}
$$

and $\mathcal{F}\left(\operatorname{LEB}_{[m, m+1)}\right)$ is $\mathcal{N}_{[m, m+1)}$-complete for every $m \in \mathbb{N}$.

Analogously, from $\mathcal{M}_{[0,1]}$-completeness of $\mathcal{F}\left(\operatorname{BAIRE}_{[0,1]}\right)$ we derive that $\mathcal{F}($ BAIRE) is $\mathcal{M}$-complete.

We can also apply Proposition 7 to mixed direct sums. For instance, we can deduce $\mathcal{J}$-completeness of $\mathcal{F}(\mathcal{S})$ where $\mathcal{J}:=\mathcal{M}_{[0,1)} \oplus \mathcal{N}_{[1,2)}$ and $\mathcal{S}:=$ $\operatorname{BAIRE}_{[0,1)} \oplus \operatorname{LEB}_{[1,2)}$.

Corollary 9. $\mathcal{F}$ (BAIRE $\cap \mathrm{LEB})$ is $\mathcal{M} \cap \mathcal{N}$-complete. 
Now, we will show that bijections preserve $\mathcal{J}$-completeness in an appriopriate way.

Proposition 10. Fix a $\sigma$-algebra $\mathcal{S}$ and $a \sigma$-ideal $\mathcal{J} \subset \mathcal{S}$ on $Y$. For any bijection $h: Y \rightarrow Z$ we have:

(a) $h * \mathcal{J}:=\{h(A): A \in \mathcal{J}\}$ is a $\sigma$-ideal and $h * \mathcal{S}:=\{h(A): A \in \mathcal{S}\}$ is a $\sigma$-algebra on $Z$.

(b) $\mathcal{F}(h * \mathcal{S})=\left\{f \circ h^{-1}: f \in \mathcal{F}(\mathcal{S})\right\}$.

(c) If $\mathcal{F}(\mathcal{S})$ is $\mathcal{J}$-complete then $\mathcal{F}(h * \mathcal{S})$ is $h * \mathcal{J}$-complete.

Corollary 11. $\mathcal{F}(\operatorname{BAIRE}(Z))$ is $\mathcal{M}(Z)$-complete for any Polish space $Z$ without isolated points. 
Let $\mathcal{S}$ and $\mathcal{J}$ be a $\sigma$-algebra and a $\sigma$-ideal on $Y$. Additionally, fix a set $X \neq \emptyset$. For $f: X \times Y \rightarrow \mathbb{R}$ and $x \in X$ we write as $(f)_{x}: Y \rightarrow \mathbb{R}$ the function given by $(f)_{x}(y):=f(x, y), y \in Y$. The set of all functions $f: X \times Y \rightarrow \mathbb{R}$ such that $(f)_{x} \in \mathcal{F}(\mathcal{S})$ for all $x \in X$ will be written as $\mathcal{F}_{X}(\mathcal{S})$.

$$
\text { If } A \subset X \times Y \text { and } x \in X \text {, we denote }(A)_{x}:=\{y \in Y:(x, y) \in A\} \text {. }
$$

We introduce two kinds of parametric convergence of $\left(f_{n}\right)_{n \in \mathbb{N}}$ to $f$ in $\mathcal{F}_{X}(\mathcal{S})$. They are defined as follows:

$$
\begin{gathered}
f_{n} \stackrel{X, \mathcal{J}}{\rightarrow} f \text { iff }(\forall x \in X)\left(f_{n}\right)_{x} \stackrel{\mathcal{J}}{\rightarrow}(f)_{x} ; \\
f_{n} \stackrel{\mathfrak{J}, X}{\longrightarrow} f \text { iff }\left(\forall\left(n_{k}\right) \in \mathbb{N}^{\uparrow}\right)\left(\exists\left(k_{p}\right) \in \mathbb{N}^{\uparrow}\right)(\forall x \in X)\left(f_{n_{k_{p}}}\right)_{x} \rightarrow(f)_{x} \text { J-a.e. on } Y . \\
\text { Of course, } f_{n} \stackrel{\mathcal{J}, X}{\rightarrow} f \text { implies } f_{n} \stackrel{X, \mathcal{J}}{\rightarrow} f . \text { The converse need not hold. }
\end{gathered}
$$


However, if $X$ is countable, then, using the diagonal technique, one can demonstrate that the both types of convergence are the same.

Example 12. We follow notation from Example 1. Consider the respective pair $(\mathcal{S}, \mathcal{J})$ on $[0,1]$ and pick $f_{n}, f \in \mathcal{F}(\mathcal{S}), n \in \mathbb{N}$, with $f_{n} \stackrel{\mathcal{J}}{\rightarrow} f$, chosen as in Example 1. Let $X:=\mathbb{N}^{\uparrow}$ and for an $x \in \mathbb{N}^{\uparrow}$ let $x=\left(m_{n}^{x}\right)_{n \in \mathbb{N}}$. Define $g_{k}: X \times[0,1] \rightarrow \mathbb{R}$, $k \in \mathbb{N}$, by putting $g_{k}(x, y):=f_{n}(y)$ if $k=m_{n}^{x}$, and $g_{k}(x, y):=0$ otherwise. Let $g(x, y):=0$ for all $(x, y) \in X \times[0,1]$. It is obvious that $\left(g_{k}\right)_{x} \stackrel{g}{\rightarrow}(g)_{x}=f$ for all $\in X$. Hence $g_{k} \stackrel{X, \mathcal{J}}{\rightarrow} g$. At the same time $g_{k} \stackrel{\mathcal{J}, X}{\rightarrow} g$ is false.

Convergence $\stackrel{\mathcal{d}, X}{\longrightarrow}$ in $\mathcal{F}_{X}(\mathcal{S})$ will be called uniform $\mathcal{J}$-convergence.

We say that a sequence $\left(f_{n}\right)_{n \in \mathbb{N}}$ in $\mathcal{F}_{X}(\mathcal{S})$ is uniformly $\mathcal{J}$-fundamental if for any $\left(m_{k}\right),\left(n_{k}\right) \in \mathbb{N}^{\uparrow}$ there is $\left(k_{p}\right) \in \mathbb{N}^{\uparrow}$ such that $\left(\left(f_{m_{k_{p}}}\right)_{x}-\left(f_{n_{k_{p}}}\right)_{x}\right)_{p \in \mathbb{N}}$ tends to zero $\mathcal{J}$-a.e. for all $x \in X$.

Every uniformly $\mathcal{J}$-convergent sequence in $\mathcal{F}_{X}(\mathcal{S})$ is uniformly $\mathcal{J}$-fundamental. 
If every uniformly $\mathcal{J}$-fundamental sequence in $\mathcal{F}_{X}(\mathcal{S})$ is uniformly $\mathcal{J}$-convergent to a function from $\mathcal{F}_{X}(\mathcal{S})$, we say that $\mathcal{F}_{X}(\mathcal{S})$ is uniformly $\mathcal{J}$-complete.

Proposition 13.(a) Let $X$ be countable. Then $\mathcal{F}_{X}(\mathcal{S})$ is uniformly $\mathcal{J}$-complete if and only if $\mathcal{F}(\mathcal{S})$ is $\mathcal{J}$-complete.

(b) Let $X_{1}$ and $X_{2}$ be equinumerable. Then $\mathcal{F}_{X_{1}}(\mathcal{S})$ is uniformly $\mathcal{J}$-complete if and only if $\mathcal{F}_{X_{2}}(\mathcal{S})$ is uniformly $\mathrm{J}$-complete.

(c) Let $\kappa, \lambda$ be cardinals. If $\lambda \geq \kappa$ and $\mathcal{F}_{\lambda}(\mathcal{S})$ is uniformly $\mathcal{J}$-complete then $\mathcal{F}_{\kappa}(\mathcal{S})$ is uniformly $\mathcal{J}$-complete.

Statement (a) can be strenghtened. Let $\mathfrak{h}$ stand for the distributivity number of $\mathcal{P}(\mathbb{N}) /[\mathbb{N}]^{<\omega}$.

Proposition 14. Let $|X|<\mathfrak{h}$. Then $\mathcal{F}_{X}(\mathcal{S})$ is uniformly $\mathcal{J}$-complete if and only if $\mathcal{F}(\mathcal{S})$ is $\mathrm{J}$-complete. 


\section{References}

- M. Balcerzak, On completeness generated by convergence with respect to a $\sigma$-ideal, Real Anal. Exchange 32 (2) (2006/2007), to appear.

- D. Vladimirov, On the completeness of partially ordered space, Uspekhi Mat. Nauk 2 (92) (1960), 165-172.

- E. Wagner, Sequences of measurable functions, Fund. Math. 112 (1981), 89-102.

- E. Wagner-Bojakowska, W. Wilczyński, Cauchy condition for the convergence in category, Proc. Amer. Math. Soc. 128 (1999), 413-418. 